Mots. Les langages du politique

\title{
Le discours présidentiel « saisi » par les séries télévisées. L'exemple du président des États-Unis
}

The presidential speech " seized " by TV series. The example of the US President El discurso presidencial « captado " por las series televisivas. El ejemplo del presidente de EE.UU

Damien Connil

\section{(2) OpenEdition}

Journals

Édition électronique

URL : https://journals.openedition.org/mots/22497

DOI : $10.4000 /$ mots. 22497

ISSN : 1960-6001

Éditeur

ENS Éditions

Édition imprimée

Date de publication : 15 octobre 2016

Pagination : 99-108

ISBN : 978-2-84788-850-8

ISSN : 0243-6450

Référence électronique

Damien Connil, «Le discours présidentiel « saisi » par les séries télévisées. L'exemple du président des États-Unis », Mots. Les langages du politique [En ligne], 112 | 2016, mis en ligne le 15 octobre 2018, consulté le 23 avril 2022. URL : http://journals.openedition.org/mots/22497 ; DOI : https://doi.org/ $10.4000 /$ mots. 22497 


\section{Damien Connil}

\section{Le discours présidentiel « saisi » par les séries télévisées. L’exemple du président des États-Unis}

House of Cards, The West Wing, Scandal, Commander in Chief, Veep, 24, The Event, State of Affairs, Homeland, Hail to the Chief, Jack \& Bobby... le président des États-Unis est régulièrement mis en scène, porté à l'écran, utilisé par la fiction : le discours présidentiel est «saisi » par les séries télévisées.

Le cinéma n'a jamais manqué de représenter, sur grand écran, le président américain. Figure tutélaire tel Abraham Lincoln chez John Ford ou Steven Spielberg $^{1}$, personnage complexe comme le Nixon d'Oliver Stone ${ }^{2}$ ou héros dans la crise, à l'exemple de Kennedy dans Treize jours3', le président à l'écran peut aussi être un personnage fictif. Tantôt sauveur de la Nation, affrontant lui-même terroristes ou extra-terrestres dans Air Force One ou Independence Day4, acteur d'un complot dans Les pleins pouvoirs ou L'Affaire Pélican, tantôt personnage qui fait sourire dans Dave voire Mars Attacks!5 ou personnage qui inquiète dans Dr Folamour ${ }^{6}$, tantôt président en devenir car candidat en campagne comme dans Primary Colors ou Les Marches du Pouvoir7, ou héros au grand cœur dans une comédie romantique comme Le Président et Miss Wade ${ }^{8}$, entre dîners officiels et protocole présidentiel, le président des États-Unis est une figure connue de la fiction.

Seulement, par la qualité de leur scénario, par la complexité de leurs personnages, par des intrigues se déroulant sur plusieurs épisodes, voire plusieurs saisons, par un rapport quotidien au spectateur, les séries télévisées ont introduit une nouvelle façon de mettre en scène le discours politique, de l’observer et de l'interroger. En s’intéressant ici à des séries télévisées prenant pour sujet la politique, on observera qu'elles participent aussi, parfois, à

1. Young Mr. Lincoln de John Ford (1939) et Lincoln de Steven Spielberg (2012).

2. Nixon de Oliver Stone (1994).

3. Thirteen days de Roger Donaldson (2000).

4. Air Force One de Wolfgang Petersen (1997) et Independence Day de Roland Emmerich (1996).

5. Absolute Power de Clint Eastwood (1997) et The Pelican Brief de Alan J. Pakula (1993).

6. Dave de Ivan Reitman (1993) et Mars Attacks! de Tim Burton (1996).

7. Primary Colors de Mike Nichols (1998) et The Ides of March de Georges Clooney (2011).

8. The American President de Rob Reiner (1995). 
la réflexion politique et entrent ainsi dans le domaine du politique (CoulombGully, Esquenazi, 2012, p.10).

Les séries télévisées sont aujourd'hui devenues de véritables objets d'étude. La littérature anglo-saxonne, pionnière, est à cet égard impressionnante. Cependant, en France aussi - et pas seulement d'ailleurs9 -, les travaux portant sur les séries télévisées prennent de l'ampleur. Les Presses universitaires de France, par exemple, leur consacrent une collection. Sans prétendre à l'exhaustivité, on remarque que des recherches sont ainsi menées aussi bien en histoire ${ }^{10}$ qu'en sociologie ou en sciences de l'information et de la communication ${ }^{11}$, aussi bien en matière d'études cinématographiques, littéraires ou anglophones s'agissant des séries américaines ${ }^{12}$ qu'en science politique ${ }^{13}$ ou en philosophie ${ }^{14}$. Ces travaux peuvent s'intéresser à la réception des fictions télévisées par leurs publics comme aux œuvres même qu'elles constituent. Dans le champ des cultural studies, dans l'interrogation des structures narratives, dans l'analyse économique des séries..., les pistes de réflexion sont nombreuses (Sepulchre, 2007).

S'agissant du discours présidentiel, les séries télévisées constituent alors de formidables outils d'appréhension et de compréhension de certains enjeux électoraux, de la fonction présidentielle elle-même et de ce qu'elle peut représenter.

\section{Du réel à la fiction : présider par la parole}

Les fictions "constituent un grand réservoir d'expériences» (Saint-Maurice, 2009, p. 9). Elles montrent ainsi, sous un jour nouveau, la «théâtralisation du politique» (Abélès, 2007, p. 41). Si, comme l'affirme Christian Le Bart, « le métier politique consiste précisément à savoir poursuivre des stratégies discursives (convaincre, se légitimer, délégitimer autrui...) en ayant intégré l'ensemble des contraintes de champ» (Le Bart, 2010, p.79), examiner comment le discours présidentiel se trouve «saisi » par la fiction est riche d'enseignements dès lors qu'aux États-Unis, «la prise de parole publique est devenue indispensable non seulement lors des campagnes électorales mais également pour exercer les fonctions présidentielles, représenter la nation, en célébrer les valeurs et faire pres-

9. Par exemple, voir, en Espagne, l'ouvrage coordonné par Pablo Iglesias et consacré aux leçons politiques de Game of Thrones, désormais traduit en français (Iglesias, 2015).

10. Notamment Boutet, 2010.

11. Citons, parmi d'autres, les travaux de Sabine Chalvon-Demersay (2015), Jean-Pierre Esquenazi (2010), Hervé Glévarec (2010) ou François Jost (2011).

12. Par exemple, Hatchuel, 2013 ou Villez, 2005.

13. Voir récemment Taïeb, 2015.

14. On pense, en particulier, aux travaux de Thibaut de Saint-Maurice (2009 et 2011), Sandra Laugier et Sylvie Allouche (2012). 
sion sur le Congrès» (Benoit à la Guillaume, 2012, p.1). Le discours présidentiel se prête alors à merveille à sa mise en scène par les séries télévisées.

Pourtant, jusqu'à la fin des années quatre-vingt-dix, rares sont les séries qui s’intéressent au président. Le tournant apparaît avec The West Wing. «Tout change en 1999 lorsque NBC et Aaron Sorkin osent faire entrer la Maison Blanche dans les foyers américains, ou inversement créent une fenêtre dans le salon des citoyens donnant directement sur le célèbre Bureau ovale» (Boutet, 2008 , p.157). Succès critique et public, la série, qui raconte le fonctionnement quotidien de l'exécutif américain en mettant en scène le président des États-Unis - un président fictif - et ses conseillers, a ouvert de nouvelles perspectives et rendu familière la parole présidentielle et, depuis, de nombreuses séries se sont emparées de la Maison Blanche sans jamais, toutefois, présenter les rouages de la présidence avec autant de précision et de détail que The West Wing. Reste que le discours présidentiel est, désormais, fréquemment porté à l'écran et mérite, pour cela au moins, que l'on s'y intéresse.

Les séries télévisées contemporaines mettent avant tout en scène les transformations les plus récentes de la présidence américaine, parmi lesquelles la question de la parole présidentielle est primordiale ${ }^{15}$. Richard Neustadt avait résumé les pouvoirs du président par une formule : «le pouvoir de persuader» (Neustadt, 1980, p. 134 et s.). L'auteur soulignait par là les limites des pouvoirs constitutionnels de la Maison Blanche, même si «les atouts d'un président sont plus importants que la simple liste de ses “pouvoirs" ne le laisserait penser» (ibid., p.137). Car, progressivement, la prééminence présidentielle s’est imposée. Le discours est devenu, pour l'hôte de la Maison Blanche, un instrument d'expression de son autorité qui lui permet de «jouer plusieurs rôles constitutionnels : chef de l'État, chef de l'exécutif, chef de la diplomatie, commandant en chef et législateur en chef» (Shogan, p.1; nous traduisons). Les séries sont ici éclairantes. Leurs épisodes montrent non seulement l'autorité du président mais aussi son rôle dans la détermination de l'agenda législatif. Les séries montrent que le discours sur l'état de l'Union - combiné avec le droit de veto qui est le seul véritable pouvoir constitutionnel du président en matière législative et que les présidents réels utilisent pour « réaliser leurs ambitions programmatiques» (Ackerman, 1998, p. 106) - permet à la Maison Blanche de jouer un rôle majeur dans l'élaboration de la loi, même si le Congrès reste maître de la procédure d’adoption. Il y a là une profonde modification de la présidence américaine ${ }^{16}$ que la fiction met parfaitement en scène.

Les discours réels des présidents des États-Unis du $20^{\mathrm{e}}$ siècle montrent, en outre, que ces derniers ne s'adressent plus seulement au Congrès mais aussi,

15. Sur ce point et pour une analyse plus détaillée dont sont issus les développements qui suivent, voir Connil, 2014.

16. En ce sens, voir l'audition de Mme E. Zoller à la Commission des lois du Sénat (France, 2008) 
directement, au peuple. Au point que «dans les années soixante, going public, c'est-à-dire la prise de parole publique pour faire pression sur le Congrès, a même supplanté le power to persuade» (Benoit à la Guillaume, 2008, p.175). Une pratique dont The West Wing se fait l'écho. Les discours du président Bartlet sont ponctués par le «nous» (we,us), caractéristique de la rhétorique moderne de la parole présidentielle (Durpaire, Snégaroff, 2008, p. 9). Plus fondamentalement, il s'adresse directement aux Américains : « We need to set our Nation on a new course, create a new history ${ }^{17}$. II leur propose de dépasser les clivages partisans et recherche directement le soutien du peuple afin de convaincre le Congrès d'adopter la législation qu'il propose. La représentation, à l'écran, du discours présidentiel met ainsi en évidence une autre transformation majeure de la présidence américaine. Pour Samuel Kernell, par exemple, le «going public» est une nouvelle stratégie du leadership présidentiel et Jeffrey Tulis parle même de «présidence rhétorique» (Kernell, 2007; Tulis, 1987).

À l'image de ce que Roland Barthes nomme "l'affiche de la Romanité» lorsqu'il observe la frange des personnages romains du Jules César de Mankiewicz (Barthes, 1957, p. 27), les séries télévisées proposent également dans leurs épisodes une forme d' " affiche de la présidentialité». Des indices visuels et même un «code visuel» (Chopin, 2002, spécialement p.52-55) permettent au spectateur de «repérer» la parole présidentielle. Pour ne prendre qu'un exemple, les discours prononcés depuis le bureau du président sont caractéristiques. La particularité du Bureau ovale renforce évidemment le code visuel, d'autant que certaines séries utilisent parfois les mêmes décors : les grandes fenêtres à petits carreaux, le bureau plutôt imposant qui rappelle celui du président Kennedy, le sceau présidentiel et, quand le président parle, un plan serré sur ce dernier avec, en arrière-plan, le drapeau des États-Unis. C'est ainsi qu'on le découvre dans The West Wing, Scandal, House of Cards, Commander in Chief, etc.

Pourtant, et presque étonnamment, le discours présidentiel lui-même est assez peu montré par les séries télévisées : «les épisodes s'achèvent alors qu'un discours présidentiel va commencer, ou débutent quand il s'éloigne déjà de la tribune. Ce sont les coulisses ou plutôt les couloirs du pouvoir exécutif, rarement visibles à l'écran, que la fiction prétend mettre au jour » (Girard, 2010, $\S 24)$. De sorte que le discours présidentiel ne renvoie pas simplement aux mots prononcés à la tribune mais aussi, beaucoup, à ce qui, en amont, en constitue la préparation et ce qui, en aval, en résultera. Le discours présidentiel saisi par la fiction présente alors cette originalité fondamentale qui est de nous montrer ce que l'on ne peut, en principe, pas voir et ainsi combler les vides de la vie politique et institutionnelle qui échappent au citoyen. Les épisodes des séries

17. «Nous devons installer notre pays sur le chemin de l'avenir en lui construisant une nouvelle histoire» (épisode 12 de la saison 6). 
télévisées dévoilent d'ailleurs certains aspects tout en faisant dire à leurs personnages que tout ne doit pas être vu : dans The West Wing, Sam Seaborn, le directeur adjoint de la communication, explique ainsi qu'il n'est pas favorable à ce qu'un article de presse soit consacré aux conseillers du président, en particulier le speechwriter car, selon lui, «les gens doivent pouvoir croire que le président écrit lui-même le discours sur l'état de l'Union » ${ }^{18}$ alors que l'épisode de la série est précisément consacré à l'élaboration et à la rédaction du discours.

Cette caractéristique est d'autant plus marquée que les séries révèlent le président des États-Unis non seulement dans l'exercice de ses fonctions, mais aussi en tant qu'être humain avec ses failles et ses faiblesses. Elles le mettent même en scène dans sa vie privée. À cet égard, Jack \& Bobby est originale. La série porte à l'écran l'adolescence, au cours des années 2000, de McAllister, président des États-Unis en 2040. Entrecoupée d'images de type documentaire et de témoignages des collaborateurs du président, elle montre que «l'on pouvait déjà reconnaître chez le jeune garçon les qualités humaines exceptionnelles qui lui permettraient par la suite d'accéder à la plus haute fonction politique» (Boutet, 2008, p.163). Par la mise en scène du discours du président, y compris dans sa vie privée, y compris dans son enfance, les séries participent d'une représentation des qualités que les citoyens attendent de leur chef d'État et participent à une forme d'imaginaire présidentiel. 24 l'illustre, par exemple, à propos de ce que les citoyens peuvent attendre du président de États-Unis en termes d'engagement, de courage et de protection de leur sécurité. Le président Palmer a l'occasion de le souligner en situation de crise. Preuve de sa magnanimité, il refuse même, à la fin de deuxième saison de la série, la démission de ceux qui ont cherché à l'écarter : «[...] je refuse votre démission. Nous avons une Nation à apaiser aujourd'hui et vous avez un rôle essentiel à jouer ${ }^{19}$.

\section{De la fiction au réel : le discours rêvé des présidents américains}

Dès lors, la fiction politique devient aussi une « arène d'interpellation de l'espace public» (Perreur, 2011, p.94). Les séries peuvent même constituer un "support de conversation et de débat » puisque "parfois indécis, ambivalents, ou au contraire trop fermes dans leurs certitudes ou préjugés, [les] personnages apprennent au gré des situations auxquelles ils sont confrontés, impliquant le téléspectateur dans leur parcours réflexif» (ibid., p. 96). Les séries peuvent ainsi interpeler leur public et mettre en débat des questions nouvelles ou interroger à nouveaux frais des questions plus classiques. Par exemple, leur

18. Épisode 11 de la saison 3.

19. Épisode 24 de la saison 2. 
traitement de la question raciale a été parfaitement mis en lumière par Olivier Esteves et Sébastien Lefait (Esteves, Lefait, 2014). S'agissant du discours présidentiel, on a pu dire que le président Palmer interprété par Dennis Haysbert dans 24 avait préparé la popularité de Barack Obama qui avait lui-même inspiré, dans The West Wing, le candidat démocrate Matt Santos, d'origine hispanique, qui succède au président Bartlet. La perspective d'une femme au pouvoir, présidente des États-Unis, apparaît également à l'écran : dans Veep, Commander in Chief, 24, State of Affairs ou, déjà, dans les années quatre-vingt, Hail to the Chief. La trajectoire politique d'Hillary Clinton inspire également les créateurs de séries. Political Animals met ainsi en scène Sigourney Weaver en ancienne First Lady nommée secrétaire d'État et Madam Secretary montre Tea Leoni en chef de la diplomatie américaine.

Les séries télévisées ne sont donc pas dépourvues de dimension politique voire idéologique, une «idéologie prime-time» selon l'expression de Mathieu de Wasseige, «une idéologie claire, parfois même preuve d'un engagement politique marqué, mais toujours contrebalancé dans une certaine mesure par des personnages, des histoires ou des pratiques contraires à cette ligne claire » (Wasseige, 2015, p. 174) comme l'illustre, par exemple, le traitement des questions de sécurité nationale et de lutte contre le terrorisme dans 24 ou dans The West Wing, les deux séries abordant, en outre, très différemment le sujet. On retrouve d'ailleurs, en ce qui concerne les séries télévisées mettant en scène le discours présidentiel, ce qui avait été observé par Erik Neveu à propos des romans d'espionnage (Neveu, 1985), à savoir parfois la conversion d'un «capital militant» en capital culturel et symbolique que certains auteurs, comme Aaron Sorkin, revendiquent.

Par la mise en scène du discours présidentiel qu'elles proposent, les séries télévisées participent, en tout état de cause, d'une «forme de connaissance, socialement élaborée et partagée [...] concourant à la construction d'une réalité commune à un ensemble social » (Jodelet, 1997, p. 53). Elles participent à la construction de représentations, d'images ou d'idées plus ou moins précises et exactes quant au discours présidentiel et à la fonction présidentielle. Cela est d'autant plus important que les séries s'appuient très largement sur la réalité, de sorte que «la part fictionnelle de la télévision n'est pas sans réagir également à des contraintes diverses exercées par l'actualité : les problèmes et autres débats de société sont une matière première presque obligatoire des séries télévisées» (Esquenazi, 2010, p. 182). Les thèmes qu'abordent les présidents fictifs font écho à ceux auxquels les présidents réels sont confrontés : éducation, contrôle des armes à feu, lutte contre le terrorisme, protection des libertés... Les séries en offrent simplement une représentation exacerbée. Dans une étude consacrée à Urgences, Sabine Chalvon-Demersay observait une représentation exagérée de la médecine d'urgence tout en soulignant «la précision technique de tout ce qui renvoie à la partie proprement médicale» 
(Chalvon-Demersay, 1999, p. 255-257). Saisir le discours présidentiel réclame pareille exigence. Cet « effet de réel» (Barthes, 1968, p. 84) fait d'ailleurs le succès des séries télévisées. Le fonctionnement des institutions doit être expliqué. Le quotidien de la Maison Blanche doit être accessible. La parole présidentielle doit être compréhensible. À défaut, la série ne convainc pas. Différents procédés explicatifs sont utilisés. Parmi eux, celui que Martin Winckler observe : "Chacun [des personnages] a, à son tour, l'occasion de se faire la voix du spectateur (du citoyen) pour dire qu'il ne comprend pas ce qui se passe, et obtenir ainsi qu'on nous/qu'on le lui explique» (Winckler, 2003). Le président peut d'ailleurs être lui-même celui qui a besoin d'explications. Dans The West Wing, face à l'obstruction parlementaire d'un sénateur, le président Bartlet avoue ne pas connaître les méandres de la procédure en vigueur au Sénat, donnant ainsi l'occasion à ses conseillers de l'éclairer ${ }^{20}$. La mise en scène du discours présidentiel permet alors au spectateur d'en saisir les enjeux politiques et institutionnels que les séries n’hésitent plus à placer au cœur de leur scénario.

L'effet de réel est encore renforcé par la «ritualité familiale» qu'offrent les séries télévisées en proposant un rendez-vous régulier, programmé, semaine après semaine, qui s'insère dans le quotidien de la vie du spectateur (Esquenazi, 2010, p.21-25). Pour mettre en scène la parole présidentielle, celles-ci s'appuient d'ailleurs très largement sur les discours dont la ritualisation institutionnelle est elle-même très forte : les discours d'investiture et les discours sur l'état de l'Union. Ces discours qui rythment la vie de la Nation (Béchillon, 2009) se trouvent ainsi particulièrement saisis par la fiction.

Mais, à l'inverse, les séries peuvent dépasser la réalité et même la prolonger. Marjolaine Boutet souligne très justement que « les premiers épisodes de The West Wing se présentent très clairement aux Américains comme ce qu'aurait pu être la politique des Démocrates au pouvoir à la fin des années quatrevingt-dix, sans le parfum de scandale et la force d'opposition d'un Congrès républicain » (Boutet, 2008, p.158). L'avènement et le succès d'une série comme House of Cards cristallisent un désenchantement à l'égard des responsables publics en même temps qu'ils témoignent d'un véritable intérêt pour le discours politique dont il convient de rendre compte. Si, à la vision idéalisée du président des États-Unis de The West Wing, s'est substituée une vision plus sombre que House of Cards ou Scandal symbolisent, il n'en demeure pas moins que le discours présidentiel se trouve désormais au cœur des séries télévisées qui inventent ainsi une nouvelle façon de parler politique.

Le discours présidentiel n'est donc pas seulement ce que dit ou ce que peut dire le président des États-Unis, mais aussi ce qu'il pourrait dire, et là se trouve - certainement - tout l'intérêt de ce qu'il soit «saisi » par la fiction. Les séries télévisées participent ainsi à la construction d’un imaginaire autour du

20. Épisode 17 de la saison 2. 
président des États-Unis qui pourrait d'ailleurs lui-même - mais c'est alors une autre question - et par un effet de retour, ne pas être sans incidence sur la réalité du discours présidentiel.

\section{Références}

ABÉlès Marc, 2007, Le spectacle du pouvoir, Paris, L'Herne.

ACKERMAn Bruce, 1998, Au nom du peuple. Les fondements de la démocratie américaine, Paris, Calmann-Lévy.

Allouche Sylvie, LAugier Sandra, 2012, Philoséries. Buffy tueuse de vampires, Paris, Bragelonne.

BARTHes Roland, 1968, «L'effet de réel », Communications, vol. 11, nº 1, p. 84-89.

- 1957, Mythologies, Paris, Seuil (Points).

BÉCHILLon Denys (de), 2009, "Congrès. La démocratie française a besoin de grandmesses institutionnelles ", La semaine juridique : édition générale, n० 85.

BENOIT À LA GUILLAUME Luc, 2008, «L'empire de la parole présidentielle. L'essor du bully pulpit au xxe siècle», L'empire de l'exécutif (1933-2006), P. Lagayette éd., Paris, PUPS, p. 175-190.

- 2012, Quand la Maison-Blanche prend la parole. Le discours présidentiel de Nixon à Obama, Berne, Berlin, Bruxelles, Peter Lang.

BOUTET Marjolaine, 2008, "Le président des États-Unis, héros de série télévisée. La figure présidentielle dans les séries américaines récentes ", Le temps des médias, no 10, p. 156-169.

- 2010, "Soixante ans d'histoire des séries télévisées américaines», Revue de recherche en civilisation américaine [En ligne], 〈http://rrca.revues.org/index248. html> (consulté le 27 mai 2016).

Chalvon-Demersay Sabine, 1999, «La confusion des sentiments. Une enquête sur la série télévisée Urgences», Réseaux, nº 95, p. 235-283.

- 2015, "Pour une responsabilité politique des héros de séries télévisées», Quaderni, n०88, p. 35-51.

CHOPIN Olivier, 2002, "La représentation des satellites dans le cinéma d'action américain », Hermès, nº 34, p. 49-63.

ConNIL Damien, 2014, "Le discours sur l'état de l'Union, The West Wing et l'imaginaire constitutionnel», Pouvoirs, nº 148, p.151-162.

Coulomb-Gully Marlène, EsquenazI Jean-Pierre, 2012, «Fiction et politique : doubles jeux», Mots. Les langages du politique, n 99, p. 5-11.

DurpaIre François, SnÉgAroff Thomas éd., 2008, L'Unité réinventée. Les présidents américains face à la nation, Paris, Ellipses.

ESqUENAZI Jean-Pierre, 2010, Les séries télévisées, l'avenir du cinéma?, Paris, Armand Colin.

Esteves Olivier, Lefalt Sébastien, 2014, La question raciale dans les séries américaines, Paris, Presses de Sciences Po.

France, SÉnAt, Commission des LoIS, 2008, audition de Mme Elizabeth Zoller devant 
la Commission des lois du Sénat, 27 mai, 〈http://www.senat.fr/compte-renducommissions/20080526/lois.html> (consulté le 27 mai 2016).

GIRARD Charles, 2010, "The world can move or not, by changing some words". La parole politique en fiction dans The West Wing", Revue de recherche en civilisation américaine [En ligne], 〈http://rrca.revues.org/index310.html〉 (consulté le 27 mai 2016).

GLEVAREC Hervé, 2010, «Trouble dans la fiction. Effets de réel dans les séries télévisées contemporaines et post-télévision », Questions de communication, n¹8, p. 214-238. HATCHUELSarah, 2013, Lost, fiction vitale, Paris, PUF.

I GLESIAS Pablo, 2015, Les leçons politiques de Game of Thrones, Paris, Post-éditions. JODELET Denise, 1997, Les représentations sociales, Paris, PUF.

Jost François, 2011, De quoi les séries américaines sont-elles le symptôme?, Paris, CNRS.

Kernell Samuel, 2007, Going Public. New Strategies of Presidential Leadership, Washington DC, CQ Press, 4 édition.

LE BART Christian, 2010, «Parler en politique», Mots. Les langages du politique, nº 94, «Trente ans d'étude des langages du politique (1980-2010) », p.77-84.

NeUSTADT Richard, 1980, Les pouvoirs de la Maison Blanche, Paris, Economica.

NeVEu Erik, 1985, L'idéologie dans le roman d'espionnage, Paris, PFNSP.

Perreur Nathalie, 2011, "La néo-série, arène d'évaluation culturelle d'une société américaine en crise ", Réseaux, n0165, p. 83-108.

SaINT-MAurice Thibaut de, 2009-2011, Philosophie en séries, Paris, Ellipses, 2 tomes.

Sepulchre Sarah, 2011, Décoder les séries télévisées, Bruxelles, De Boeck.

Shogan Colleen J., 2015, "The President's State of the Union Address: tradition, function, and policy implications », Congressional Research Service, R 40132, 16 janvier.

TAḮB Emmanuel, 2015, «House of Cards. Qu'est-ce qu'un coup politique fictionnel? », Quaderni, n०88, p.67-81.

Tulis Jeffrey, 1987, The Rhetorical Presidency, Princeton, Princeton University Press.

VILLEz Barbara, 2005, Séries télé : visions de la justice, Paris, PUF.

WASSEIGE Mathieu (de), avec la collaboration de DuPONT Barbara, 2015, Séries télé US:

l'idéologie prime time, Louvain-la-Neuve, Academia-L'Harmattan.

WinCKLer Martin, 2003, "La Maison Blanche en série TV», Le Monde Diplomatique, septembre; repris sous le titre "The West Wing / À la Maison Blanche» et désormais disponible sur 〈http://martinwinckler.com/article.php3?id_article=39〉 (consulté le 27 mai 2016). 
